\title{
Application of Segawa-Suzuki Spectral Mapping Theorem
}

\author{
Hong Ji, Caishi Wang, Nan Fan
}

\begin{abstract}
In this paper, we consider a class of unitary operators associated with the evolution operators of the quantum walk . By using Segawa and Suzuki's spectral mapping theorem, we obtain their spectra.
\end{abstract}

Keywords-Quantum Bernoulli noises; Spectral mapping theorem; Operators

\section{INTRODUCTION}

As quantum analogs of classical random walks, quantum walks have drawn great attention in recent years. One typical feature of a quantum walk is that it usually has a ballistic spread speed compared to the diffusion spread speed of a classical random walk. Quantum walks have found wide application in quantum information and quantum computation. Due to the quantum interference effects, quantum walks greatly outperform random walks at certain computational tasks, and moreover it has turned out that quantum walks constitute universal models of quantum computation.

From a perspective of mathematics, a time -homogeneous quantum walk is usually described by a unitary operator on a complex Hilbert space and a unit vector in the same Hilbert space. Conventionally, the unitary operator is known as the walk's evolution operator, while the unit vector is referred to as its initial state. Given its initial state, a quantum walk is then completely determined by its evolution operator.

In [4], the authors introduced a model of time-inhomogeneous quantum walk in terms of quantum Bernoulli noises and showed that its evolution operators are unitary involutions (self-adjoint unitary operators). On the other hand, in their recent paper [2], Segawa and Suzuki have proven a spectral mapping theorem for a class of abstract quantum walks.

In this paper, we consider a class of unitary operators associated with the evolution operators of the quantum walk introduced in [4], and, by using Segawa and Suzuki's spectral mapping theorem mentioned above, we obtain their spectra.

\section{PRELIMINARIES}

In this section, we first recall some necessary notions and facts about quantum Bernoulli noises,

and then we describe the Segawa and Suzuki's spectral mapping theorem.

\subsection{Quantum Bernoulli noises}

Let $\Omega$ be the set of all functions $\omega: \square \mapsto\{-1,1\}$, and $\left(\zeta_{n}\right)_{n \geq 0}$ the sequence of canonical projections on $\Omega$. Let $F$ be the $\sigma$-field on $\Omega$ generated by the sequence $\left(\zeta_{n}\right)_{n \geq 0}$, and $\left(p_{n}\right)_{n \geq 0}$ a given sequence of positive numbers with the property that $0<p_{n}<1$ for all $n \geq 0$. Then there exists a unique probability measure $P$ on the measurable space $(\Omega, F)$ such that

$P \circ\left(\zeta_{n_{1}}, \zeta_{n_{2}}, \ldots, \zeta_{n_{k}}\right)^{-1}\left\{\left(\varepsilon_{1}, \varepsilon_{2}, \ldots, \varepsilon_{k}\right)\right\}=\prod_{j=1}^{k} p_{n_{j}} \frac{1+\varepsilon_{j}}{2}\left(1-p_{n_{j}}\right)^{\frac{1-\varepsilon_{j}}{2}}$ for $\quad n_{j} \in N, \varepsilon_{j} \in\{-1,1\}(1 \leq j \leq k) \quad$, with $n_{i} \neq n_{j}$ when $i \neq j$ and $k \geq 1$. Thus one has a probability measure space $(\Omega, \mathrm{F}, \mathrm{P})$, which is referred to as the Bernoulli space and complex-valued random variables on it are known as Bernoulli functionals.

Let $Z=\left(Z_{n}\right)_{n \geq 0}$ be the sequence of Bernoulli functionals generated by the sequence, $\left(\zeta_{n}\right)_{n \geq 0}$ namely

$$
Z_{n}=\frac{\zeta_{n}+q_{n}-p_{n}}{2 \sqrt{p_{n} q_{n}}}, \quad \mathrm{n} \geq 0,
$$

where $q_{n}=1-p_{n}$. Clearly $Z=\left(Z_{n}\right)_{n \geq 0}$ is a sequence of independent random variables on the probability measure $\operatorname{space}(\Omega, F, P)$. Let $\hbar$ be the space of square integrable Bernoulli functionals, namely

$$
\hbar=L^{2}(\Omega, \quad \mathrm{F},)
$$

We denote by $\langle\cdot, \cdot\rangle$ the usual inner product of the space $\hbar$, and by $\|\cdot\|$ the corresponding norm. It is known that $Z$ has the chaotic representation property, which implies that $\mathfrak{I}=\left\{Z_{\tau} \mid \tau \in \Gamma\right\}$ forms an orthonormal basis (ONB) for $\hbar$, which is known as the 
canonical ONB for $\hbar$. Here $Z_{\varnothing}=1$ and

$$
Z_{\tau}=\prod_{j \in \tau} Z_{j}, \quad \tau \in \Gamma, \quad \tau \neq \varnothing .
$$

Clearly $\hbar$ is infinite-dimensional as a complex Hilbert space and, for $n \geq 0, Z_{n}=Z_{\{n\}}$ is a member of the canonical ONB for $\hbar$.

It can be shown that [3], for each $k \geq 0$, there exists a bounded operator $\partial_{k}$ on $\hbar$ such that

$\partial_{k} Z_{\tau}=1_{\tau}(k) Z_{\tau \backslash k}, \quad \partial_{k}^{*} Z_{\tau}\left[1-1_{\tau}(k)\right] Z_{\tau \cup k}, \quad \tau \in \Gamma$, wher $\partial_{k}^{*}$ denotes the adjoint of $\partial_{k}, \tau \backslash k=\tau \backslash\{k\}$, and $1_{\tau}(k)$ the indicator of $\tau$ as a subset of $\square$.

The operators $\partial_{k}$ and $\partial_{k}^{*}$ are usually known as the annihilation and creation operators acting on Bernoulli functionals, respectively. And the family $\left\{\partial_{k}, \partial_{k}^{*}\right\}_{k \geq 0}$ is referred to as quantum Bernoulli noises.

A typical property of quantum Bernoulli noises is that they satisfy the canonical anti-commutation relations (CAR) in equal-time [3]. More specifically, for $k, l \geq 0$, it holds true that

$\partial_{k} \partial_{l}=\partial_{l} \partial_{k}, \partial_{k}^{*} \partial_{l}^{*}=\partial_{l}^{*} \partial_{k}^{*}, \partial_{k}^{*} \partial_{l}=\partial_{l} \partial_{k}^{*}, \quad(k \neq l)$ and

$$
\partial_{k} \partial_{k}=\partial_{k}^{*} \partial_{k}^{*}=0, \partial_{k} \partial_{k}^{*}+\partial_{k}^{*} \partial_{k}=I,
$$

where $I$ is the identity operator on $\hbar$.

\subsection{Segawa-Suzuki spectral mapping theorem}

Definition 2.1. Let $\mathbf{H}$ be a Hilbert space. A unitary operator $S$ on $\mathbf{H}$ is called a unitary involution on $\mathrm{H}$ if it is self-adjoint.

Clearly, a bounded operator $S$ on a Hilbert space $\mathrm{H}$ is a unitary involution if and only if it satisfies $S^{*}=S$ and $S^{2}=I$. Here $I$ means the identity operator on $\mathrm{H}$. If $P$ is a projection operator on Hilbert space $\mathrm{H}$, then $2 P-I$ is a unitary involution.

Definition 2.2. Let $H, \mathrm{~K}$ be Hilbert spaces. A bounded operato $A: H \rightarrow K$ is called a coisometry form $\mathrm{H}$ to $K$ if it satisfies $A A^{*}=I_{k}$. Here $I_{k}$ means the identity operator on $K$.

Let $A$ be a coisometry form Hilbert space $\mathrm{H}$ to Hilbert space $K$. Then it can be shown that $2 A^{*} A-I_{H}$ is a unitary involution on $\mathrm{H}$. By con- vention, we usually use the simpler $2 A^{*} A-1$ to mean $2 A^{*} A-I_{H}$.

Lemma 2.1. [2] Let $\boldsymbol{H}, \mathrm{K}$ be complex Hilbert spaces. Suppose that $S$ is a unitary involution on $\mathrm{H}$ and $d_{A}$ is a coisometry form $\mathrm{H}$ to $K$. Write $U=S\left(2 d_{A}^{*} d_{A}-1\right)$ and $T=d_{A} S d_{A}^{*}$. Then it holds true that

$$
\begin{aligned}
& \sigma(U)=\varphi^{-1}(\sigma(T)) \cup\{1\}^{M_{+}} \cup\{-1\}^{M_{-}}, \\
& \sigma_{p}(U)=\varphi^{-1}\left(\sigma_{p}(T)\right) \cup\{1\}^{M_{+}} \cup\{-1\}^{M_{-}},
\end{aligned}
$$

where $M_{ \pm}=\operatorname{dim}\left[\operatorname{ker} d_{A} \cap \operatorname{ker}(S \pm 1)\right]$ and $\varphi$ is the Joukowsky transform given by

$$
\varphi(x)=\frac{x+x^{-1}}{2} .
$$

Moreover, for $\lambda \in \sigma_{p}(U)$, one has

$\operatorname{dim} \operatorname{ker}(U-\lambda)= \begin{cases}\operatorname{dim} \operatorname{ker}(T-\varphi(\lambda)), & \lambda \neq \pm 1 ; \\ \operatorname{dim} \operatorname{ker}(T \mp 1)+M_{ \pm}, & \lambda= \pm 1,\end{cases}$

Under the conditions in Lemma 2.1, the operator $U=S\left(2 d_{A}^{*} d_{A}-1\right)$ is called the abstract evolution associated with $S$ and $d_{A}$, while the operator $T=d_{A} S d_{A}^{*}$ is called the discriminant of $U$. As is seen, Lemma 2.1 actually gives a spectral mapping theorem between an abstract evolution and its discriminant.

\section{MAIN RESULTS}

In this section, we state and prove our main results. We first make some necessary preparations.

Let $l^{2}(\square, \hbar)$ be the space of square summable functions defined on $\square$ and valued in $\hbar$, namely

$$
l^{2}(\square, \hbar)=\left\{\Phi: \square \mapsto \hbar \mid \sum_{x=-\infty}^{x=\infty}\|\Phi(x)\|^{2}<\infty\right\}
$$

where $\|\cdot\|$ means the norm of $\hbar$. Then $l^{2}(\square, \hbar)$ remains a complex Hilbert space, whose inner product $\langle\cdot, \cdot\rangle_{l^{2}(\square, \hbar)}$ is given by

$$
\langle\Phi, \Psi\rangle_{l^{2}(\square, \hbar)}=\sum_{x=-\infty}^{x=\infty}\langle\Phi(x), \Psi(x)\rangle, \Phi, \Psi \in l^{2}(\square, \hbar),
$$

where $\langle\cdot, \cdot\rangle$ denotes the inner product of $\hbar$ as indicated in Subsection 2.1. By convention, we denote by 
$\|\cdot\|_{l^{2}(\square, \hbar)}$ the norm induced by $\langle\cdot, \cdot\rangle_{l^{2}(\square, \hbar)}$. As usual, a vector $\Phi \in l^{2}(\square, \hbar)$ is called normalized if $\|\Phi\|_{l^{2}(\square, \hbar)}=1$. For $z \in \square$ and $u \in \hbar$, we use $\phi_{z \text {, u }}$ to mean the $\hbar$-valued function on $\square$ given by

$$
\phi_{z, \mathrm{u}}= \begin{cases}u, & \mathrm{x}=\mathrm{z} ; \\ 0, & \mathrm{x} \in \square, \mathrm{x} \neq \mathrm{z} .\end{cases}
$$

Clearly, $\phi_{z, \mathrm{u}} \in l^{2}(\square, \hbar)$ and $\left\|\phi_{z, \mathrm{u}}\right\|_{l^{2}(\square, \hbar)}=\|u\|$ for all $z \in \square$ and $u \in \hbar$.

Lemma 3.1. [4] For each nonnegative integer $k \geq 0$, there exists a unitary involution $U_{k}$ on $l^{2}(\square, \hbar)$ such that $\left[U_{k} \Phi\right](x)=\partial_{k} \Phi(x-1)+\partial_{k}^{*} \Phi(x+1), \quad \mathrm{x} \in \square, \Phi \in 1^{2}(\square, \hbar)$.

In [4], actually by using the operators $\left\{U_{k} \mid k \geq 0\right\}$ as the evolution operators, the authors introduced a model of time-inhomogeneous quantum walk and showed its basic properties. In the following, we analyze a class of unitary operators associated with $\left\{U_{k} \mid k \geq 0\right\}$.

Proposition 3.2. Define a mapping $P: l^{2}(\square, \hbar)$

$\rightarrow \hbar$ as $P \Phi=\Phi(0), \quad \Phi \in 1^{2}(\square, \hbar)$. Then $P$ is coisometry from $l^{2}(\square, \hbar)$ to $\hbar$, and moreover its adjoint $P^{*}$ satisfies $P^{*} u=\phi_{0, \mathrm{u}}, \mathrm{u} \in \hbar$.

Proof. It is easy to see that $P$ is a linear operator from $l^{2}(\square, \hbar)$ to $\hbar$. For all $\Phi \in l^{2}(\square, \hbar)$, a simple calculation gives

$$
\|P \Phi\|=\|\Phi(0)\| \leq\|\Phi\|_{l^{2}(\square, \hbar),}
$$

which implies that $P$ is bounded. Now let $u \in \hbar$ be given. Then, for all $\Phi \in l^{2}(\square, \hbar)$, we have

$$
\begin{gathered}
\left\langle P^{*} u, \Phi\right\rangle_{l^{2}(\square, \hbar)}=\langle u, \mathrm{P} \Phi\rangle=\langle u, \Phi(0)\rangle \\
=\sum_{x \in \square}\left\langle\varnothing_{0, \mathrm{u}}(x), \Phi(x)\right\rangle=\left\langle\varnothing_{0, \mathrm{u}}, \Phi\right\rangle_{l^{2}(\square, \hbar)} .
\end{gathered}
$$

which means $P^{*} u=\phi_{0, \mathrm{u}}$. Finally, for all $u \in \hbar$, by using the above property of $P^{*}$ as well as properties $P P^{*} u=P \phi_{0, \mathrm{u}}=\phi_{0, \mathrm{u}}(0)=u$, of $\phi_{0, \mathrm{u}}$, we find

$$
P P^{*} u=P \phi_{0, \mathrm{u}}=\phi_{0, \mathrm{u}}(0)=u,
$$

which implies $P P^{*}=I_{\hbar}$, namely $P$ is a coisometry from $l^{2}(\square, \hbar)$ to $\hbar$.

As is mentioned above, if $A$ is a bounded operator on $\hbar$, then we conventionally use $A-1$ to mean $A-I_{\hbar}$.

Proposition 3.3. Let $k \geq 0$ be a nonnegative integer and write $W_{k}=U_{k}\left(2 P^{*} P-1\right)$. Then $W_{k}$ is a unitary operator on $l^{2}(\square, \hbar)$.

Proof. Since $U_{k}$ is a unitary operator, it suffices to show that $2 P^{*} P-1$ is unitary. In fact, by the property $P P^{*}=I_{\hbar}$, we have

$\left(2 P^{*} P-1\right)^{2}=4 P^{*} P P^{*} P-4 P^{*} P+1=4 P^{*} P-4 P^{*} P+1=1$, which together with $\left(2 P^{*} P-1\right)^{*}=2 P^{*} P-1$ implies that $2 P^{*} P-1$ is unitary. Here 1 means the identity operator on $\hbar$.

In what follows, we focus on analyzing the unitary operators $\left\{W_{n} \mid n \geq 0\right\}$ from a perspective of the spectral theory. For brevity, we always assume that $k \geq 0$ is a fixed nonnegative integer. Recall that $T_{k}=P U P^{*}$, which is called the discriminant of $W_{k}$. The next proposition shows that it is actually a null operator on $\hbar$.

Theorem 3.4. It holds true that $T_{k}=0$. In particular, $\sigma\left(T_{k}\right)=\sigma_{p}\left(T_{k}\right)=\{0\}$.

Proof. Let $u \in \hbar$ be given. Then, $P^{*} u=\phi_{0, \mathrm{u}}$, which together with Proposition 3.2 and Lemma 3.1 gives

$T_{k} u=P U_{k} P^{*} u=P\left[U_{k \phi_{0, \mathrm{u}}}\right](0)=\partial_{k \phi_{0, \mathrm{u}}}(-1)+\partial_{k \phi_{0, \mathrm{u}}}^{*}(1)=0$. Here properties of the function $\phi_{0, \mathrm{u}}$ is used. Thus, $T_{k}=0$ as an operator on $\hbar$, which naturally implies $\sigma\left(T_{k}\right)=\sigma_{p}\left(T_{k}\right)=\{0\}$.

The next theorem is our main result, which gives the spectrum and point spectrum of the unitary operator $W_{k}$.

Theorem 3.5. The operator $W_{k}$ has a spectrum and point spectrum of the following form $\sigma\left(W_{k}\right)=\sigma_{p}\left(W_{k}\right)=\{i,-i\} \cup\{1\}^{M_{+}} \cup\{-1\}^{M_{-}}$. where $\quad M_{ \pm}=\operatorname{dim}\left[\operatorname{ker} P \cap \operatorname{ker}\left(U_{k} \pm 1\right)\right] \quad$ and 
$\{ \pm 1\}^{M_{ \pm}}$that \pm 1 is the eigenvalue of multiplicity $M_{ \pm}$. And moreover, one has

$\operatorname{dim} \operatorname{ker}\left(W_{k} \pm i\right)=+\infty, \quad \operatorname{dim} \operatorname{ker}\left(W_{k} \pm 1\right)=M_{\text {干. }}$ Proof. Recall that $W_{k}=U_{k}\left(2 P^{*} P-1\right)$ and $T_{k}=P U P^{*}$, where, by Lemma 3.1, $U_{k}$ is a unitary involution and, by Proposition 3.2, $P$ is a coisometry.

Hence, using Lemma 2.1, we have

$$
\sigma\left(W_{k}\right)=\varphi^{-1}\left(\sigma\left(T_{k}\right)\right) \cup\{1\}^{M_{+}} \cup\{-1\}^{M_{-}}
$$

with $M_{ \pm}=\operatorname{dim}\left[\operatorname{ker} P \cap \operatorname{ker}\left(U_{k} \pm 1\right)\right]$, where $\varphi$ is the Joukowsky transform given by

$$
\varphi(x)=\frac{x+x^{-1}}{2} .
$$

This together with Theorem 3.4 as well as the equality $\varphi^{-1}(\{0\})=\{i,-i\}$ gives

$\sigma\left(W_{k}\right)=\varphi^{-1}(\{0\}) \cup\{1\}^{M_{+}} \cup\{-1\}^{M_{-}}=\{i,-i\} \cup\{1\}^{M_{+}} \cup\{-1\}^{M_{-}}$.

Similarly, we can verify

$$
\sigma_{p}\left(W_{k}\right)=\{i,-i\} \cup\{1\}^{M_{+}} \cup\{-1\}^{M_{-}} \text {. Again using }
$$

Lemma 2.1, we have

$$
\operatorname{dim} \operatorname{ker}\left(W_{k}-\lambda\right)= \begin{cases}\operatorname{dim} \operatorname{ker}\left(T_{k}-\varphi(\lambda)\right), & \lambda= \pm i ; \\ \operatorname{dim} \operatorname{ker}\left(T_{k} \mp 1\right)+M_{ \pm}, & \lambda= \pm 1,\end{cases}
$$

which, together with Theorem 3.4 as well as the equalities $\varphi( \pm i)=0$ and $\hbar=+\infty$, implies above .

\section{ATCKNOWLEDGEMENT}

This work is supported by National Natural Science Foundation of China (Grant No. 11861057).

\section{REFERENCE}

[1] N. Privault, Stochastic analysis of Bernoulli processes, Probability Surveys 5 (2008), 435-483.

[2] E. Segawa and A. Suzuki, Spectral mapping theorem of an abstract quantum walk,Quantum Information Processing 18 (2019), Paper No. 333.

[3] C.S. Wang, H.F Chai and Y.C. Lu, Discrete-time quantum Bernoulli noises, \{lit J. Math. Phys. $\}\{$ lbf 51\} (2010), no. 5, 053528.

[4] Y. Zhang, C.S. Wang, J. Shi and L.X. Zhang, A unitary quantum walk constructed directly from quantum Bernoulli noises, International Journal of Engineering and Applied Sciences 6 (2019), Issue 9, 57-60.

Hong Ji, School of Mathematics and Statistics, Northwest Normal
University, Lanzhou, China, Mobile, 86-15117199615.

Caishi Wang, School of Mathematics and Statistics, Northwest Normal University, Lanzhou, China.

Nan Fan, School of Mathematics and Statistics, Northwest Normal University, Lanzhou, China. 